\title{
Metastatic neuroendocrine carcinoma of the colon: response to standard colorectal therapy
}

\author{
Francisco Acevedo $\cdot$ Claudia Otarola $\cdot$ \\ Jose Valbuena $\cdot$ Marcelo Garrido
}

Received: 10 August 2012/Accepted: 8 October 2012/Published online: 15 November 2012

(C) The Japan Society of Clinical Oncology 2012

\begin{abstract}
Colorectal neuroendocrine carcinoma (NEC) is a rare disease. There are no prospective randomized studies evaluating the best approach for these patients. Colorectal NEC is frequently treated in a similar way to small-cell cancers, because of its histologic appearance. We report a case of metastatic colorectal NEC which was treated with standard chemotherapy for metastatic colonic adenocarcinoma, including biological agents. A 47-year-old woman, with an unremarkable past medical history, presented to the emergency room complaining of a five-day history of abdominal distension, diffuse crampy pain, and vomiting. An abdominal computed tomography scan showed an obstructive tumor in the descending colon associated with multiple focal hepatic lesions. On the same day, she underwent an emergency laparotomy. A histologic examination revealed a tumor composed of malignant small and medium-sized cells with scant cytoplasm and immunoreactivity to chromogranin and synaptophysin consistent with a diagnosis of NEC. Six out of 20 excised lymph nodes were compromised, and a biopsy of the hepatic lesion showed similar features to the primary tumor. She later received
\end{abstract}

F. Acevedo $(\varangle) \cdot$ M. Garrido

Departamento de Hematología-Oncología, Facultad de

Medicina, Pontificia Universidad Catolica de Chile,

Diagonal Paraguay 319, Santiago, Chile

e-mail: fnacevedo@gmail.com

C. Otarola

Departamento de Radiologia, Facultad de Medicina,

Pontificia Universidad Catolica de Chile,

Diagonal Paraguay 319, Santiago, Chile

J. Valbuena

Departamento de Anatomia Patologica, Facultad de Medicina,

Pontificia Universidad Catolica de Chile, Diagonal Paraguay

319, Santiago, Chile palliative chemotherapy with 5-fluorouracil/leucovorin plus oxaliplatin (FOLFOX) and bevacizumab every other week, completing six cycles. At the end of the treatment she showed clinical improvement and partial response by imaging studies without significant toxicities. We emphasize the report on the treatment received by this patient and the need for further investigation on this matter.

Keywords Colorectal $\cdot$ Neuroendocrine $\cdot$ Neoplastic . Therapy

\section{Introduction}

Colorectal neuroendocrine carcinoma (NEC) is a rare disease. There are no prospective randomized studies to evaluate the best approach for these patients. Colorectal NEC is frequently treated in a similar way to small-cell cancers, because of its histologic appearance. We report a case of metastatic colorectal NEC treated with standard chemotherapy for metastatic colonic adenocarcinoma, including biological agents.

\section{Case report}

A 47-year-old woman, with an unremarkable past medical history, presented to the emergency department because of a five-day history of progressive abdominal distension, biliary vomiting, diffuse crampy pain, along with an inability to pass stool and flatus. She also reported significant weight loss and anorexia over the previous few months. However, no medical advice was sought at that time. Her arterial pressure, temperature, and cardiac frequency were normal. Physical examination revealed a distended 

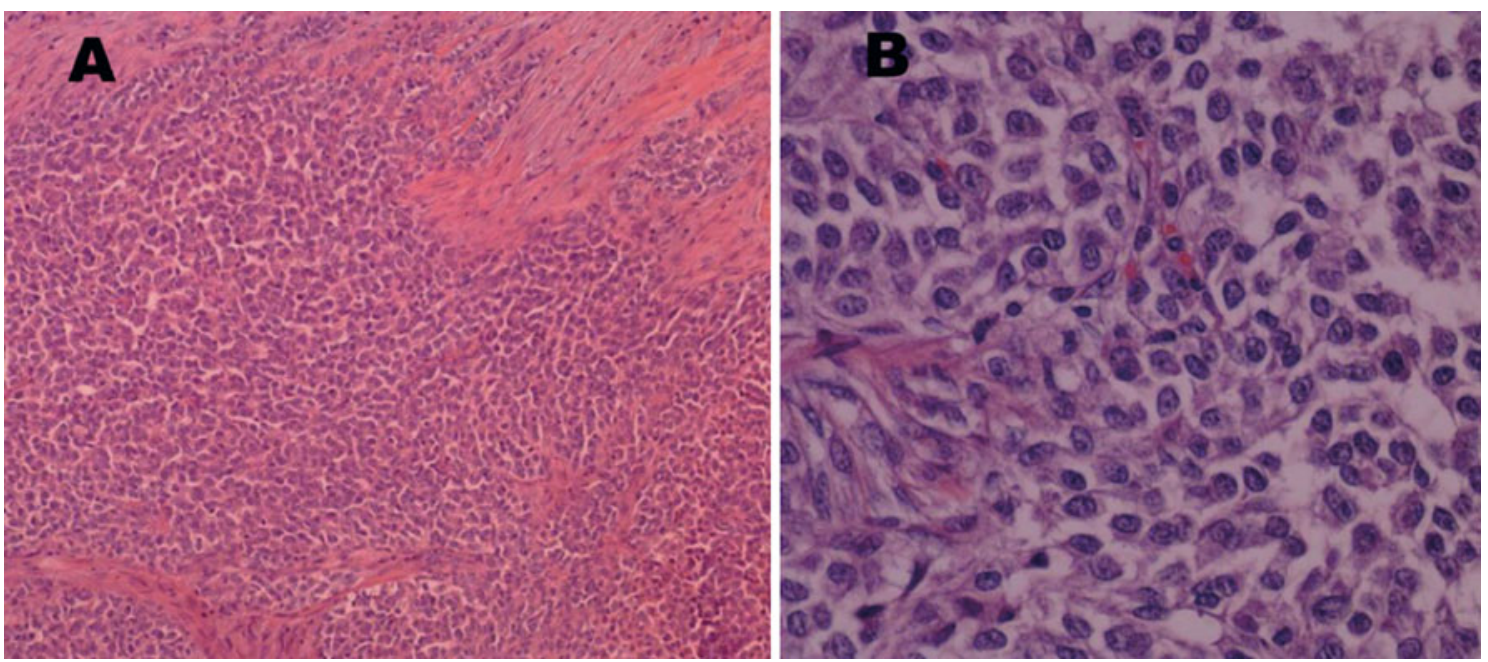

Fig. 1 a Histologic examination of the surgical specimen with hematoxylin-eosin. b Tumor was mainly composed of small and medium-sized cells with scant cytoplasm, small nuclei, and granular chromatin

abdomen without bowel sounds. Tenderness and rebound were also present.

A hemogram revealed mild anemia with a hemoglobin level of $9 \mathrm{mg} / \mathrm{dL}$ and normal white blood and platelet counts. Renal and liver functions were within the normal range, and her carcinoembryonic antigen (CEA) level was $0.7 \mathrm{ng} / \mathrm{mL}$.

An abdominal computed tomography scan (CT) showed a tumor arising from the descending colon causing intestinal obstruction associated with multiple focal hepatic lesions. On the same day, an emergency left hemicolectomy with primary anastomosis and a regional lymphadenectomy was performed.

Macroscopically, the tumor corresponded to an irregular and annular constricting mass of size $5 \times 2 \times 6 \mathrm{~cm}$ which arose from the mucosa infiltrating the pericolonic adipose tissue. A histologic examination revealed a tumor composed of malignant small and medium-sized cells with scant eosinophilic cytoplasm and small nuclei with granular chromatin (Fig. 1). No gland formation was evident in these areas, and the mitotic rate was low with two figures per ten high-power fields (HPFs). The Ki-67 proliferative index was $30 \%$ in the tumor cells (Fig. 2). Immunohistochemical staining resulted in positive results for pancytokeratin, neuron-specific enolase, chromogranin A, and synaptophysin, consistent with a diagnosis of NEC (Fig. 3). Six out of 20 excised lymph nodes were compromised, and a biopsy of the hepatic lesion showed similar features to those in the colon.

Further distant extension was examined with a thorax CT scan and a radionuclide bone scan, which showed no evidence of metastasis to other sites.

She had no complications during the postoperative period, and was discharged in order to complete treatment on an ambulatory basis. Six weeks after the surgery, being

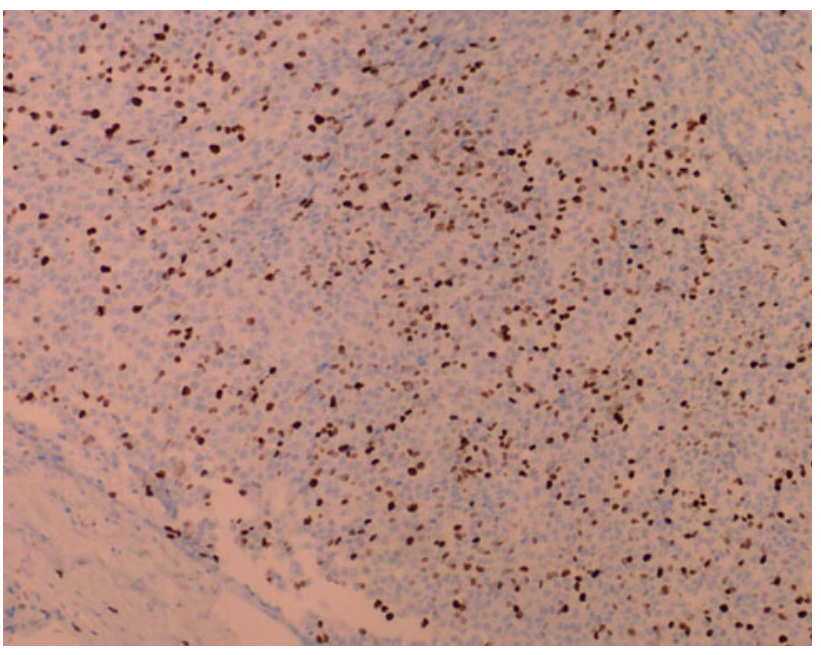

Fig. 2 The proliferation fraction, as demonstrated by nuclear immunoreactivity to the antigen $\mathrm{Ki}-67$, was $30 \%$ in the tumor cells

a patient with ECOG performance status 1, she received polychemotherapy with 5-fluorouracil/leucovorin plus oxaliplatin (FOLFOX)-4 (5-fluorouracil $400 \mathrm{mg} / \mathrm{m}^{2}$ bolus followed by a $1,200 \mathrm{mg} / \mathrm{m}^{2} 44 \mathrm{~h}$ infusion; leucovorin $200 \mathrm{mg} / \mathrm{m}^{2}$ days $1-2$; and oxaliplatin $85 \mathrm{mg} / \mathrm{m}^{2}$ on day 1 ) plus bevacizumab at $5 \mathrm{mg} / \mathrm{kg}$ every other week, completing six cycles without significant toxicities.

The patient was evaluated during the third and sixth months of the treatment, and she showed a partial tumor response according to the Response Evaluation Criteria in Solid Tumors (RECIST v.1.1) (Fig. 4). The clinical evolution was also favorable, as her performance status recovered to ECOG 0 early in the first month of treatment. Side effects were mild, with only grade 2 neuropathy according to the Common Terminology Criteria for Adverse Events (CTCAE, v. 4.0). Neither febrile neutropenia nor delays in 

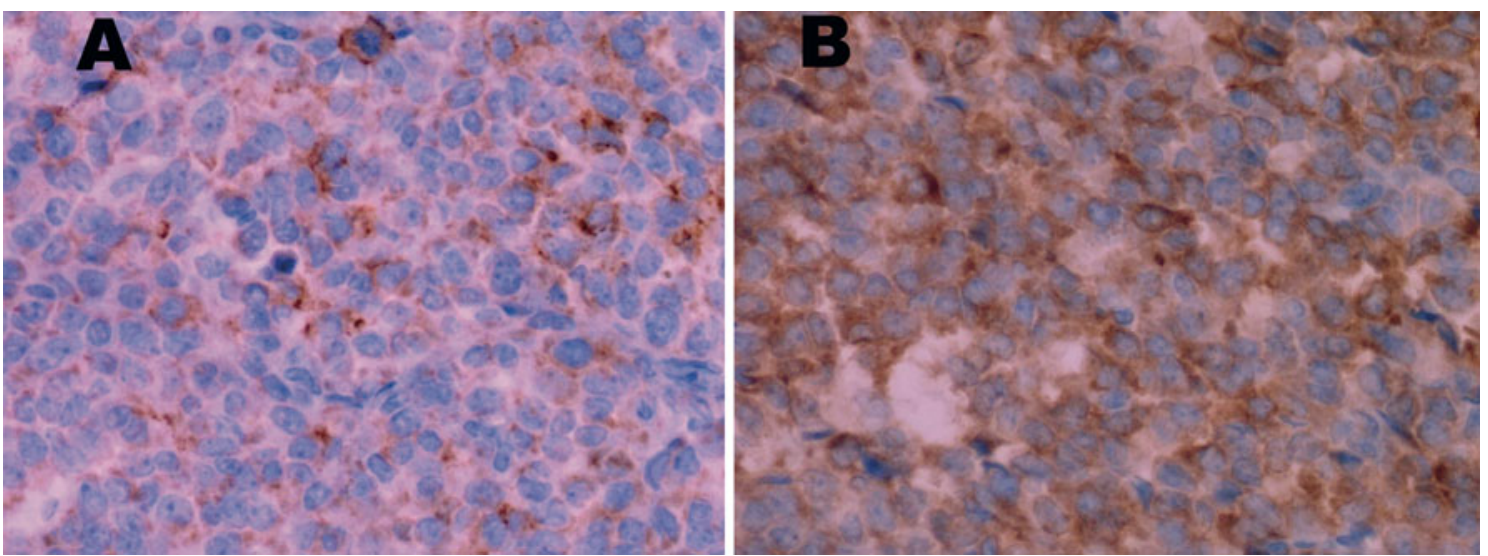

Fig. 3 Immunohistochemical staining resulted in positive results for CD56 (a) and synaptophysin (b), consistent with a diagnosis of neuroendocrine carcinoma

Fig. 4 a Abdominal CT displaying multiple metastatic liver lesions in a patient with colonic cancer (primary not shown). b Evaluation after six cycles of chemotherapy with FOLFOX shows a partial response according to the RECIST criteria
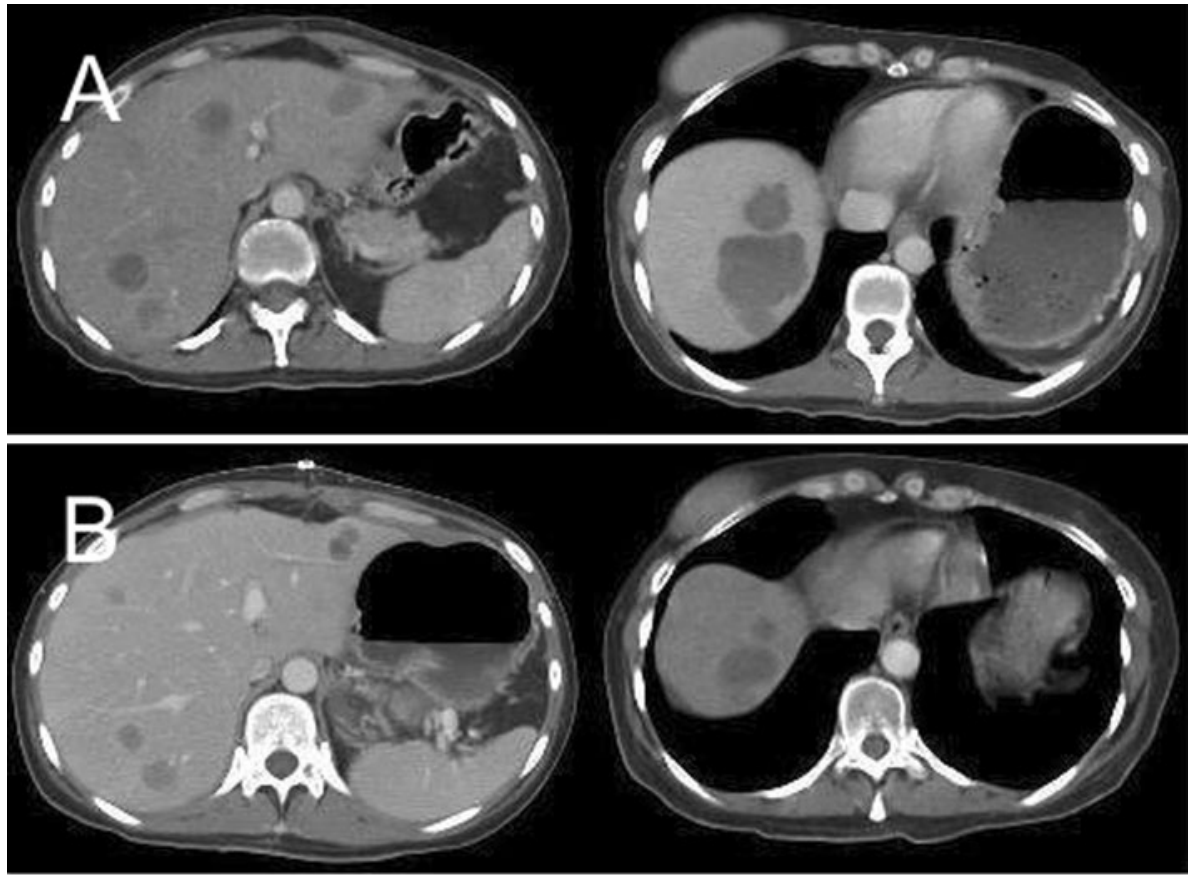

chemotherapy administration were noted. She did not have any reported episodes of hypertension, proteinuria, or thromboembolic disease associated with the use of bevacizumab. Three months after the last evaluation, progression of the liver metastasis was noticed, so she started second-line chemotherapy with cisplatin $\left(100 \mathrm{mg} / \mathrm{m}^{2}\right)$ and etoposide $\left(100 \mathrm{mg} / \mathrm{m}^{2}\right)$ but showed no response after three cycles. She died 13 months after diagnosis.

\section{Discussion}

Colorectal cancer is a common disease. More than one million new cases are reported every year worldwide, with a mortality rate of nearly 8.1 per 100,000 population. It is the third most commonly diagnosed cancer in males, and the second in females [1]. There is significant variation in its incidence around the globe, with the highest rates occurring in highly developed countries such as Australia and the United States. However, recent reports have demonstrated that several newly developed nations are presenting even higher incidence rates, probably due to the adoption of known environmental risk factors, in a process called "Westernization" [2]. On the other hand, death rates have declined progressively in many Western countries, mainly due to earlier detection enabled by more effective screening programs and the use of treatments incorporating biological agents such as bevacizumab and cetuximab.

In Chile, colorectal carcinoma is the third highest cause of mortality due to gastrointestinal cancer after gastric 
cancer and biliary neoplasm. Although mortality trends analysis has demonstrated that colorectal cancer mortality is either decreasing or stabilizing in many developed and newly developed countries, data from our nation shows a significant rising tendency from 1993 to 2003 [2, 3].

In spite of its relatively good prognosis overall, metastatic colorectal carcinoma is present in approximately $19 \%$ of patients, with survival rates of only $8.1 \%$ at five years [4]. In this setting, the use of systemic chemotherapy along with targeted therapy has become the firstline treatment, yielding a median survival time of almost 25 months [5, 6]. An exception occurs in patients who are considered to have resectable metastatic disease, who can potentially be cured after local and systemic therapy [7].

Histologically, more than $97 \%$ of all colorectal cancers are adenocarcinomas, and approximately $10 \%$ of them present focal neuroendocrine differentiation, with some reports suggesting that this finding is an independent prognostic factor in stages III and IV [8, 9]. The remaining colorectal cancers are rare histologic types, among which, according to the literature, between 0.3 and $3.9 \%$ are NEC [10-12].

The pathological classification of these tumors has been controversial over the years. There are some epidemiological reports suggesting that poorly differentiated endocrine carcinoma may have been misdiagnosed as adenocarcinoma in the previous century [13]. It is very important to note the wide spectrum of this disease, which ranges from low-grade carcinomas such as carcinoids to high-grade carcinomas with poorly differentiated tumors like NEC or small cell carcinoma. Our case was a moderately differentiated pure neuroendocrine tumor, which is categorized between carcinoid and small cell undifferentiated carcinoma. In relation to prognosis, reports of this variety show an ominous prognosis, resembling the small cell variant [14].

Primary colonic NECs occur mainly in the right colon. Clinically, they are no different from adenocarcinomas, and no specific risk factor has been linked with their development. Nevertheless, they are highly aggressive malignancies with very poor prognosis, and $70 \%$ of patients have metastatic disease at the time of diagnosis [11]. According to Kang et al. [10], they have the worst overall survival rates of all colorectal neoplasms. Only $5 \%$ of patients with the metastatic disease are alive at 5 years [9].

There are no prospective randomized studies evaluating the best approach for these patients. Besides its rareness, the exact nomenclature used for the disease is controversial, which means that this disease is underrepresented in clinical trials. Surgical resection is performed in most localized disease cases. In advanced disease, systemic chemotherapy is the recommended approach, as in any colorectal cancer. Unfortunately, the best drug or combination of them has not been established for this histological type of carcinoma.

The histogenesis of this neoplasm is uncertain. The most widely accepted theory, based on the usual association with glandular or squamous precursors, suggests that a primitive neuroendocrine cell serves as a common precursor for all types of gastrointestinal neoplasm [15]. Nevertheless, there are some authors who have demonstrated some genetic changes similar to those that occur in colorectal carcinoma [16]. According to the Vogelstein multistep process of carcinogenesis, some germline or somatic mutations in colorectal cancer are required for malignant transformation [17]. There is evidence that neuroendocrine tumors share some of these genetic changes (loss of function of APC, DCC, and p53), suggesting an "adenoma-neuroendocrine" sequence [18]. Moreover, classical carcinoids do not share these pathways, thus indicating a genetically distinct neoplasm.

Mismatch repair genes are responsible for recognizing and repairing small insertions or deletions that occur during DNA replication. Germline mutation in one of these genes is the main genetic defect in patients with hereditary nonpolyposis colorectal cancer, but loss of the expression of these genes can also be found in $15 \%$ of sporadic colorectal cancer [19].

A retrospective study of 39 colorectal carcinomas with neuroendocrine features ( 27 were NECs) demonstrated the presence of microsatellite instability (MSI), which is characteristic of mismatch repair deficiency, in $15 \%$ of the cases [20].

However, these types of observations were not reproduced in a report which found that MSI was an infrequent event in poorly differentiated colorectal neuroendocrine tumors compared to sporadic colorectal cancer [18].

Regarding tumor growth and invasiveness, angiogenesis plays a central role. Different molecules mediate this process; one of them is the vascular endothelial growth factor (VEGF). It is known that VEGF mRNA and protein are upregulated in colorectal cancer, and that its overexpression is associated with poor prognosis [21]. In this way, bevacizumab, a humanized monoclonal antibody directed against VEGF, has been shown to improve overall survival in metastatic colorectal carcinoma [5, 6], and is currently part of its standard treatment. In one study, patients with poorly differentiated colorectal carcinoma and neuroendocrine cell differentiation showed higher VEGF protein expression levels and microvessel densities than patients without neuroendocrine differentiation [21]. The opposite has also been demonstrated, in that neuroendocrine digestive tumors express fewer genes and proteins related to neoangiogenesis than adenocarcinomas.

There is no standard treatment for metastatic colorectal NECs. Due to its histologic appearance, NECs are 
Table 1 Comparison of different schemes of chemotherapy used for neuroendocrine colorectal carcinoma

\begin{tabular}{|c|c|c|c|}
\hline Reference & $\begin{array}{l}\text { Number } \\
\text { of patients }\end{array}$ & Treatment & $\begin{array}{l}\text { Overall } \\
\text { survival } \\
\text { (months) }\end{array}$ \\
\hline $\begin{array}{l}\text { Saclarides } \\
\text { et al. [11] }\end{array}$ & 39 & $\begin{array}{l}\text { Cyclophosphamide, } \\
\text { vincristine, } \\
\text { doxorubicin }\end{array}$ & $5-7$ \\
\hline Bernick et al. [12] & 38 & $\mathrm{P}, \mathrm{EP}, 5 \mathrm{FU} / \mathrm{LV}$ & 10.5 \\
\hline Moertel et al. $[22]^{\mathrm{a}}$ & 45 & $\mathrm{EP}$ & $10.5-19$ \\
\hline $\begin{array}{l}\text { Villalonga } \\
\text { et al. [23] }\end{array}$ & 5 & EP/5FU-STZ & - \\
\hline Pape et al. $[24]^{\mathrm{a}}$ & 16 & FOLFOX & $\begin{array}{l}\text { TTP } \\
18 \text { weeks }\end{array}$ \\
\hline $\begin{array}{c}\text { Cheng-Chou } \\
\text { et al. [25] }\end{array}$ & 11 & $\begin{array}{c}\text { EP, 5FU/LV, } \\
\text { FOLFIRI }\end{array}$ & 14 \\
\hline
\end{tabular}

$P$ cisplatin, $E P$ etoposide-cisplatin, $5 F U$ 5-fluorouracil, $L V$ leucovorin, $S T Z$ streptozocin

${ }^{\text {a }}$ Include neuroendocrine carcinoma of other sites

frequently treated in a similar way to small-cell cancers of the lung, with cytotoxic regimens including cisplatin and/or etoposide, or in a similar manner to pancreatic neuroendocrine tumor, with anthracycline, which is not usually used in the treatment of colonic adenocarcinomas (Table 1). However, because of some biological similarities with colorectal carcinoma (see above), drugs like oxaliplatin, capecitabine, and 5FU have been used, and have yielded objective responses and survivals similar to other regimens (Table 1). We decided to use this treatment in our case because the healthcare system in our country only covers standard systemic treatment that is approved for colorectal carcinoma.

Administration of biological agents is rare, with only one report (to our knowledge) describing its use with standard colonic treatment, which achieved a complete clinical response [26].

Predictive factors for the treatment of NEC with standard chemotherapy, including biologic agents are lacking. Further studies are necessary to elucidate the effectiveness of this approach.

Conflict of interest The authors declare that they have no conflict of interest.

\section{References}

1. Jemal A, Bray F, Center MM et al (2011) Global cancer statistics. CA Cancer J Clin 61:69-90

2. Center M, Ahmedin J, Smith R et al (2009) Worldwide variations in colorectal cancer. CA Cancer J Clin 59:366-378

3. Donoso A, Villarroel L, Pinedo G (2006) Increase in colon cancer mortality rates in Chile, during the period 1990-2003 (in Spanish). Rev Med Chile 134:152-158
4. O'Connell JB, Maggard MA, Ko CY (2004) Colon cancer survival rates with the American Joint Committee on Cancer sixth edition staging. J Natl Cancer Inst 96:1420-1425

5. Hurwitz H, Fehrenbacher L, Novotny W et al (2004) Bevacizumab plus irinotecan, fluorouracil, and leucovorin for metastatic colorectal cancer. N Engl J Med 350:2335-2342

6. Hochster HS, Hart LL, Ramanathan RK et al (2008) Safety and efficacy of oxaliplatin and fluoropyrimidine regimens with or without bevacizumab as first-line treatment of metastatic colorectal cancer: results of the TREE Study. J Clin Oncol 26: 3523-3529

7. Morris EJ, Forman D, Thomas JD et al (2010) Surgical management and outcomes of colorectal cancer liver metastases. Br J Surg 97:1110-1118

8. Grabowski P, Sturm I, Schelwies K et al (2006) Analysis of neuroendocrine differentiation and the p53/BAX pathway in UICC stage III colorectal carcinoma identifies patients with good prognosis. Int J Colorectal Dis 21:221-230

9. Grabowski P, Schindler I, Anagnostopoulos I et al (2001) Neuroendocrine differentiation is a relevant prognostic factor in stage III-IV colorectal cancer. Eur J Gastroenterol Hepatol 13:405-411

10. Kang H, O'Connell JB, Leonardi MJ et al (2007) Rare tumors of the colon and rectum: a national review. Int J Colorectal Dis 22:183-189

11. Saclarides TJ, Szeluga D, Staren ED (1994) Neuroendocrine cancers of the colon and rectum. Dis Colon Rectum 37:635-642

12. Bernick PE, Klimstra DS, Shia J et al (2004) Neuroendocrine carcinomas of the colon and rectum. Dis Colon Rectum 47:163-169

13. Ahlman H, Nilsson O, McNicol AM et al (2007) Poorly differentiated endocrine carcinomas of midgut and hindgut origin. Neuroendocrinology 87:40-46

14. Gaffey M, Mills S, Lack E (1990) Neuroendocrine carcinoma of the colon and rectum. Am J Surg Pathol 14:1010-1023

15. Ho KJ, Herrera GA, Jones JM et al (1984) Small cell carcinoma of the esophagus: evidence for a unified histogenesis. Hum Pathol $15: 460-468$

16. Vortmeyer AO, Lubensky IA, Merino MJ et al (1997) Concordance of genetic alterations in poorly differentiated colorectal neuroendocrine carcinomas and associated adenocarcinomas. J Natl Cancer Inst 89:1448-1453

17. Fearon ER, Vogelstein B (1990) A genetic model for colorectal tumorigenesis. Cell 61:759-767

18. Arnold CN, Nagasaka T, Goel A et al (2008) Molecular characteristics and predictors of survival in patients with malignant neuroendocrine tumors. Int J Cancer 123:1556-1564

19. Shibata D, Peinado MA, Ionov Y et al (1994) Genomic instability in repeated sequences is an early somatic event in colorectal tumorigenesis that persists after transformation. Nat Genet 6:273-281

20. La Rosa S, Marando A, Furlan D et al (2012) Colorectal poorly differentiated neuroendocrine carcinomas and mixed adeno neuroendocrine carcinomas: insights into the diagnostic immunophenotype, assessment of methylation profile, and search for prognostic markers. Am J Surg Pathol 36:601-611

21. Ishigami SI, Arii S, Furutani M et al (1998) Predictive value of vascular endotelial growth factor (VEGF) in metastasis and prognosis of human colorectal cancer. Br J Cancer 78:1379-1384

22. Moertel CG, Kvols LK, O'Connell MJ et al (1991) Treatment of neuroendocrine carcinomas with combined etoposide and cisplatin. Evidence of major therapeutic activity in the anaplastic variants of these neoplasms. Cancer 68:227-232

23. Villalonga R, Espin Basany E, Lopez Cano M (2008) Neuroendocrine carcinomas of the colon and rectum. A unit's experience over six years. Rev Esp Enferm Dig 100:11-16 
24. Pape U, Tiling C, Bartel U et al (2006) Oxaliplatin plus 5-fluorouracil/folinic acid as palliative treatment for progressive malignant gastrointestinal neuroendocrine carcinomas. ASCO Annu Meet Proc Part I(24):14074

25. Cheng-Chou L, Chih-Wei W, Chung-Rong C et al (2008) Neuroendocrine carcinomas of the colon and rectum: result of a
http://www.crs.org.tw/upload_newsletter/9710/71_04-R97012.pdf. Accessed Sept 2012

26. Yabuki H, Suto T, Inoue $\mathrm{K}$ et al (2012) A case of colorectal neuroendocrine carcinoma effectively treated with bevacizumab + levofonilate + 5-FU chemotherapy (in Japanese). Gan To Kagaku Ryojo 39:1139-1142 